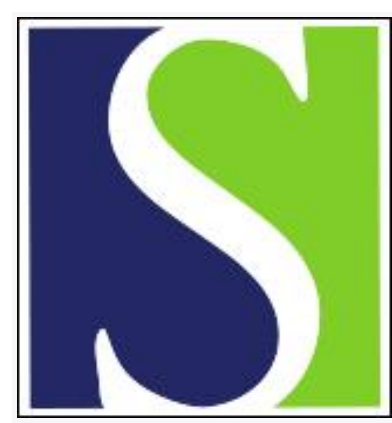

Scand J Work Environ Health 1988;14(6):344-355

https://doi.org/10.5271/sjweh.1908

Issue date: Dec 1988

Methodological considerations in the study of parental occupational exposures and congenital malformations in offspring.

by Shaw GM, Gold EB

Affiliation: California Birth Defects Monitoring Program, California Department of Health Services, Emeryville 94608.

This article in PubMed: www.ncbi.nlm.nih.gov/pubmed/3062770

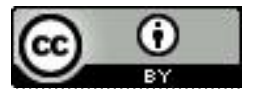




\title{
Methodological considerations in the study of parental occupational exposures and congenital malformations in offspring
}

\author{
by Gary M Shaw, DrPH, ${ }^{1}$ Ellen B Gold, $\mathrm{PhD}^{1,2}$
}

\begin{abstract}
SHAW GM, GOLD EB. Methodological considerations in the study of parental occupational exposures and congenital malformations in offspring. Scand J Work Environ Health 14 (1988) 344-355. The existence of hazardous substances in the workplace has raised concerns about the potential of these substances for adverse reproductive effects. Identification of associations between parental occupational exposures and congenital malformations in the offspring may provide the opportunity for preventing such exposures and thus reduce the risk of malformations. However, there are many methodological considerations inherent in studying the potential relation between parental occupational exposures and congenital malformations in the offspring. Considerations relating to outcome include methods and timing of ascertaining cases with malformations, diagnostic criteria, and problems in grouping malformations for purposes of analysis. With regard to measuring exposures, issues include methods for obtaining valid estimates of the nature, duration, timing of exposure, and exposure-response relationship. Other methodological issues discussed include selection of appropriate reference groups, sample size, and multiple hypothesis testing.
\end{abstract}

Key terms: epidemiologic methods, pregnancy outcome, review.

In North America congenital malformations account for about $20 \%$ of infant mortality (9). They are the second leading cause of death among children aged one to four years and the third leading cause among children aged 15 to 19 years. Most congenital malformations are believed to be due to the interaction of both environmental and genetic influences. Currently, approximately $20 \%$ of human malformations can be ascribed to genetic transmission, $5 \%$ to chromosome aberrations, and approximately $10 \%$ to all known environmental factors, leaving approximately $65-70 \%$ of all malformations with no known or suspected cause (87).

Several reviews of the literature have provided detailed accounts of the few suspected risk factors for malformations and have reviewed some of the issues specific to the epidemiology of congenital malformations $(39,42,44)$. Our objective in this review was to address the many methodological considerations inherent in studies of the potential relation between parental occupational exposure and congenital malformations among offspring. The justification for studying a possible relation between a parent's exposure at work and subsequent development of a congenital malformation in his or her child is based on the notion

1 California Birth Defects Monitoring Program, California Department of Health Services and Health Officers Association of California, Berkeley, California, the United States.

2 Division of Occupation and Environmental Medicine, University of California, Davis, California, the United States.

Reprint requests to: Dr GM Shaw, California Birth Defects Monitoring Program, 5900 Hollis Street, Suite A, Emeryville, CA 94608, USA. that exposures to potential hazards may affect parental germ cells before conception or may affect embryonic somatic cells after conception; either action may induce cell death or dysfunction leading to malformations in the offspring (31). The importance of epidemiologic studies of relevant occupational exposures lies in the potential for preventing congenital malformations if risks associated with such exposures are identified and exposures are reduced or eliminated.

\section{Considerations related to congenital malformations}

\section{Biological considerations}

The teratogenic effect of a specific occupational exposure is unlikely to result in a general increase in all types of malformations although it may increase the risk of more than one malformation, particularly if the exposure occurs during a specific time of gestation when more than one organ system is developing. In table 1 several examples are given of investigations which involved all malformations grouped together rather than specific malformations as the end point of interest $(5,6,62)$. A generic problem for studies of malformations is whether to group malformations. From a biological perspective, it is important not to group congenital malformations into a single outcome but rather to consider them as heterogeneous outcomes which may have distinct etiologies. The choice of a specific malformation or group of malformations for study should be based upon embryologic information about which organs and tissues are developing at the time of exposure. In addition, decisions about which 
Table 1. Selected studies of parental occupational exposures and congenital malformations. (BDMP = birth defect monitoring program, CLP = cleft lip and palate, CNS = central nervous system, GI = gastrointestinal, MACDP = Metropolitan Atlanta Congenital Defect Program, MS $=$ musculoskeletal, NAAA $=$ National Agricultural Aviation Association, $O R=0 d d s$ ratio, $\mathrm{PVC}=$ polyvinyl chloride, $\mathrm{RR}=$ relative risk, VDT = video display terminal)

\begin{tabular}{|c|c|c|c|c|c|c|c|}
\hline Reference & Subjects & $\begin{array}{l}\text { Study } \\
\text { period }\end{array}$ & $\begin{array}{l}\text { Source of } \\
\text { occupational } \\
\text { information }\end{array}$ & $\begin{array}{l}\text { Timing of } \\
\text { exposure }\end{array}$ & $\begin{array}{l}\text { Maternal/ } \\
\text { paternal } \\
\text { exposure }\end{array}$ & $\begin{array}{l}\text { Length of } \\
\text { ascertain- } \\
\text { ment of mal- } \\
\text { formations }\end{array}$ & Results \\
\hline \multicolumn{8}{|c|}{ Laboratory chemicals } \\
\hline $\begin{array}{l}\text { Holmberg (34) } \\
\text { and Holmberg \& } \\
\text { Hernberg (35) }\end{array}$ & $\begin{array}{l}\text { Cases: CNS defects, Finnish } \\
\text { Register ( } N=120) \\
\text { Referents: preceding delivery } \\
\text { in same maternal welfare } \\
\text { district ( } N=120 \text { ) } \\
\text { Second paper added oral } \\
\text { clefts to the cases and their } \\
\text { referents }(N=102 \text { pairs) }\end{array}$ & $1976-1977$ & $\begin{array}{l}\text { Interview } \\
\text { with } \\
\text { mothers }\end{array}$ & $\begin{array}{l}\text { First } \\
\text { trimester } \\
\text { Any time } \\
\text { during } \\
\text { pregnancy }\end{array}$ & Maternal & $\begin{array}{l}\text { First year } \\
\text { postnatal }\end{array}$ & $\begin{array}{l}\text { Matched } O R=13 / 2 \text { for } \\
\text { organic solvents } \\
(P<0.01) ; \\
\text { OR }=1.4 \text { for parental } \\
\text { manufacture jobs and } \\
C N S ; O R=1.3 \text { for } \\
\text { community service } \\
\text { and clefts }\end{array}$ \\
\hline $\begin{array}{l}\text { Hansson et al } \\
\text { (27) }\end{array}$ & $\begin{array}{l}\text { Exposed: Swedish chemical } \\
\text { laboratory workers' infants } \\
(\mathrm{N}=103) \\
\text { Unexposed: other pharma- } \\
\text { ceutical employees' infants } \\
(\mathrm{N}=297)\end{array}$ & $\begin{array}{l}\text { Not } \\
\text { stated }\end{array}$ & $\begin{array}{l}\text { Postal } \\
\text { questionnaire }\end{array}$ & Not stated & Maternal & At birth & $\begin{array}{l}6 / 103 \text { exposed vs } 2 / 297 \\
\text { unexposed had major } \\
\text { malformations }(R R=8.6)\end{array}$ \\
\hline $\begin{array}{l}\text { Ericson \& } \\
\text { Kallen (23) }\end{array}$ & $\begin{array}{l}\text { Cases: esophageal, anal or } \\
\text { small bowel atresia }(N=201) \\
\text { Referents: from Swedish birth } \\
\text { register, no Gl atresia, two per } \\
\text { case, matched on maternal } \\
\text { age, parity, month of deliv- } \\
\text { ery, completed triplets }=142\end{array}$ & $1976-1978$ & $\begin{array}{l}\text { Physician or } \\
\text { social worker } \\
\text { interviewed } \\
\text { parent of } \\
\text { cases \& mal- } \\
\text { formation } \\
\text { referents; } \\
\text { postal ques- } \\
\text { tionnaire to } \\
\text { normals }\end{array}$ & $\begin{array}{l}\text { During } \\
\text { pregnancy }\end{array}$ & Maternal & $\begin{array}{l}\text { Neonatal } \\
\text { period }\end{array}$ & $\begin{array}{l}\text { OR }=3 \text { for parental } \\
\text { laboratory work } 4.3 \% \text { of } \\
\text { cases, } 1.9 \% \text { expected, } \\
1.4 \% \text { of referents } \\
(P<0.01) ; \\
\text { matched triplets } O R=6\end{array}$ \\
\hline $\begin{array}{l}\text { Leffingwell } \\
\text { et al }(49)\end{array}$ & $\begin{array}{l}\text { Exposed: female laboratory } \\
\text { workers of reproductive age } \\
\text { in a pharmaceutical } \\
\text { company }(\mathrm{N}=164) \\
\text { Unexposed: female clerical! } \\
\text { administrative workers in } \\
\text { the same company }(\mathrm{N}=318)\end{array}$ & $1968-1977$ & $\begin{array}{l}\text { Company } \\
\text { records }\end{array}$ & $\begin{array}{l}\text { At concep- } \\
\text { tion and/or } \\
\text { during preg- } \\
\text { nancy }\end{array}$ & Maternal & $\begin{array}{l}\text { Not } \\
\text { stated }\end{array}$ & $\begin{array}{l}\text { Excess of malformations } \\
(\mathrm{N}=6) \text { among laboratory } \\
\text { workers in } 1970 \mathrm{~s} \text {, particu- } \\
\text { larly for laboratory work } \\
\text { at time of conception; } \\
\text { none in administrative } \\
\text { workers }\end{array}$ \\
\hline Olsen (60) & $\begin{array}{l}\text { Cases: CNS malformations } \\
\text { or malformations of the gut, } \\
\text { extremities and CLP - } \\
\text { Funen County, Denmark } \\
(N=619)\end{array}$ & $1972-1976$ & $\begin{array}{l}\text { Birth } \\
\text { certificate }\end{array}$ & At birth & $\begin{array}{l}\text { Maternal/ } \\
\text { paternal }\end{array}$ & $\begin{array}{l}\text { Not } \\
\text { stated }\end{array}$ & $\begin{array}{l}\text { OR }=1.9 \text { for CNS malfor- } \\
\text { mations and unskilled } \\
\text { father; OR }=2.8 \text { for CNS } \\
\text { malformations and pater- } \\
\text { nal organic solvent ex- } \\
\text { posure }(95 \% \mathrm{Cl} 0.8-9.4) \text {; } \\
\mathrm{OR}=4.9 \text { for } \mathrm{CNS} \text { malfor- } \\
\text { mations and painter } \\
\text { father; no association of } \\
\text { paternal or maternal } \\
\text { laboratory assistant and } \\
\text { anomalies of gut or ex- } \\
\text { tremities or clefts }\end{array}$ \\
\hline $\begin{array}{l}\text { Axelsson et al } \\
\text { (2) }\end{array}$ & $\begin{array}{l}\text { Exposed: women exposed } \\
\text { to solvents in laboratory - } \\
\text { University of Göteborg } \\
\text { ( } N=492 \text { pregnancies) } \\
\text { Unexposed: women not ex- } \\
\text { posed to solvents in labora- } \\
\text { tory work ( } N=496 \text { pregnan- } \\
\text { cies) }\end{array}$ & $1968-1979$ & $\begin{array}{l}\text { Postal } \\
\text { question- } \\
\text { naire }\end{array}$ & $\begin{array}{l}\text { First } \\
\text { trimester } \\
\text { and re- } \\
\text { mainder of } \\
\text { pregnancy }\end{array}$ & Maternal & $\begin{array}{l}\text { Neonatal } \\
\text { period }\end{array}$ & $\begin{array}{l}3.7 \% \text { malformations } \\
\text { among exposed; } 4.2 \% \\
\text { among unexposed }\end{array}$ \\
\hline $\begin{array}{l}\text { Ericson et al } \\
\text { (22) }\end{array}$ & $\begin{array}{l}\text { Cases: serious malformations } \\
\text { in children of Swedish } \\
\text { laboratory workers }(\mathrm{N}=24) \\
\text { Referents: two per case, } \\
\text { stratified on age and parity, } \\
\text { laboratory workers }(\mathrm{N}=48)\end{array}$ & 1975 & $\begin{array}{l}\text { Census data } \\
\text { and postal } \\
\text { questionnaire }\end{array}$ & $\begin{array}{l}\text { During } \\
\text { pregnancy }\end{array}$ & Maternal & $\begin{array}{l}\text { Neonatal } \\
\text { period }\end{array}$ & $\begin{array}{l}\text { No difference in working } \\
\text { in laboratory during preg- } \\
\text { nancy; increase in malfor- } \\
\text { mations over expected } \\
\text { from population }\end{array}$ \\
\hline $\begin{array}{l}\text { Kurppa et al } \\
\text { (47) }\end{array}$ & $\begin{array}{l}\text { Cases: CNS, heart, skeletal, } \\
\text { oral cleft malformations in } \\
\text { Finnish Register }(N=1047) \\
\text { Referents: preceding deliv- } \\
\text { ery in same district }\end{array}$ & $\begin{array}{l}\text { Not } \\
\text { stated }\end{array}$ & $\begin{array}{l}\text { Interview } \\
\text { and } \\
\text { industrial } \\
\text { hygienist }\end{array}$ & $\begin{array}{l}\text { First } \\
\text { trimester }\end{array}$ & Maternal & $\begin{array}{l}\text { First year } \\
\text { of life }\end{array}$ & $\begin{array}{l}\text { Maternal exposure to or- } \\
\text { ganic solvents about } \\
\text { twice as high for CNS } \\
\text { and three times higher } \\
\text { for oral clefts than for } \\
\text { referents }\end{array}$ \\
\hline $\begin{array}{l}\text { Holmberg \& } \\
\text { Nurminen }(36)\end{array}$ & $\begin{array}{l}\text { Cases: CNS defects (primar- } \\
\text { ily anencephaly) in Finnish } \\
\text { Register }(N=120) \\
\text { Referents: preceding delivery } \\
\text { in same district }(N=120)\end{array}$ & $1976-1978$ & Interview & $\begin{array}{l}\text { First } \\
\text { trimester }\end{array}$ & $\begin{array}{l}\text { Maternal/ } \\
\text { paternal }\end{array}$ & $\begin{array}{l}\text { First year } \\
\text { of life }\end{array}$ & $\begin{array}{l}\text { OR }=5.5 \text { for maternal ex- } \\
\text { posure to organic solvents } \\
\text { in first trimester }(\mathrm{P}<0.025) \\
\mathrm{OR}=3.4 \text { for dust ex- } \\
\text { posure }(\mathrm{P}<0.01) \text {; dust and } \\
\text { solvents multiplicative; } \\
\text { no difference for metals, } \\
\text { pesticides, dyes, noise, } \\
\text { temperature, radiation, or } \\
\text { safety equipment use }\end{array}$ \\
\hline
\end{tabular}

(continued) 
Table 1. Continued.

\begin{tabular}{|c|c|c|c|c|c|c|c|}
\hline Reference & Subjects & $\begin{array}{l}\text { Study } \\
\text { period }\end{array}$ & $\begin{array}{l}\text { Source of } \\
\text { occupational } \\
\text { information }\end{array}$ & $\begin{array}{l}\text { Timing of } \\
\text { exposure }\end{array}$ & $\begin{array}{l}\text { Maternal/ } \\
\text { paternal } \\
\text { exposure }\end{array}$ & $\begin{array}{l}\text { Length of } \\
\text { ascertain- } \\
\text { ment of mal- } \\
\text { formations }\end{array}$ & Results \\
\hline \multicolumn{8}{|l|}{ Anesthetics } \\
\hline $\begin{array}{l}\text { Knill-Jones } \\
\text { et al }(\mathbf{4 5 )}\end{array}$ & $\begin{array}{l}\text { Exposed: married female } \\
\text { anesthetists in United King- } \\
\text { dom ( } N=563) \\
\text { Unexposed: married female } \\
\text { physicians (nonanesthetists) } \\
\text { from medical register in } \\
\text { United Kingdom }(N=828)\end{array}$ & 1970 & $\begin{array}{l}\text { Postal } \\
\text { question- } \\
\text { naire }\end{array}$ & $\begin{array}{l}\text { First and } \\
\text { second } \\
\text { trimester }\end{array}$ & Maternal & $\begin{array}{l}\text { Not } \\
\text { stated }\end{array}$ & $\begin{array}{l}\text { Malformation frequency of } \\
\text { offspring of anesthestists } \\
\text { working during first six } \\
\text { months of pregnancy } 6.7 \% \\
\text { compared to } 2.5 \% \text { for } \\
\text { anesthestists not working }\end{array}$ \\
\hline $\begin{array}{l}\text { Corbett et al } \\
\text { (16) }\end{array}$ & $\begin{array}{l}\text { Exposed: practicing Michigan } \\
\text { nurse anesthetists }(\mathrm{N}=434) \\
\text { Unexposed: nonpracticing } \\
\text { nurse anesthetists and } \\
\text { nonanesthetists }(\mathrm{N}=261)\end{array}$ & $\begin{array}{l}\text { Not } \\
\text { stated }\end{array}$ & $\begin{array}{l}\text { Postal } \\
\text { question- } \\
\text { naire }\end{array}$ & $\begin{array}{l}\text { During } \\
\text { pregnancy }\end{array}$ & Maternal & Childhood & $\begin{array}{l}16.4 \% \text { birth defects } \\
\text { among exposed and } \\
5.7 \% \text { among unexposed } \\
\text { ( } P<0.005 \text { ); largest differ- } \\
\text { ences in skin (especially } \\
\text { hemangiomas) and MS } \\
\text { defects (especially ingui- } \\
\text { nal hernia) }\end{array}$ \\
\hline $\begin{array}{l}\text { Pharoah et al } \\
\text { (65) }\end{array}$ & $\begin{array}{l}\text { Exposed: Medical Register } \\
\text { women who practiced } \\
\text { anesthesia during preg- } \\
\text { nancy in England and } \\
\text { Wales ( } N=670 \text { pregnancies) } \\
\text { Unexposed: pregnancy con- } \\
\text { ceived during other practice } \\
\text { ( } N=6377 \text { ) }\end{array}$ & $1950-1975$ & $\begin{array}{l}\text { Postal } \\
\text { question- } \\
\text { naire }\end{array}$ & $\begin{array}{l}\text { At } \\
\text { conception }\end{array}$ & Maternal & Childhood & $\begin{array}{l}\text { Malformations of heart } \\
\text { and greater vessels in off- } \\
\text { spring of anesthetists } \\
(13.8 / 1000 \text { vs } 3.6 / 1000 \text {, } \\
P<0.05)\end{array}$ \\
\hline $\begin{array}{l}\text { Ericson \& } \\
\text { Kallen (23) }\end{array}$ & $\begin{array}{l}\text { Exposed: Swedish women } \\
\text { working in operating room } \\
\text { ( } \mathrm{N}=494 \text { infants) } \\
\text { Unexposed: women working } \\
\text { in medical work ( } \mathrm{N}=19127 \\
\text { infants) }\end{array}$ & $\begin{array}{l}1973 \& \\
1975\end{array}$ & Records & $\begin{array}{l}\text { During } \\
\text { pregnancy }\end{array}$ & Maternal & $\begin{array}{l}\text { Not } \\
\text { stated }\end{array}$ & $\begin{array}{l}\text { No significant difference } \\
\text { in malformations }(4.5 \% \\
\text { vs } 5.2 \%)\end{array}$ \\
\hline $\begin{array}{l}\text { Cohen et al } \\
\text { (14) }\end{array}$ & $\begin{array}{l}\text { Exposed: American dentists } \\
\text { and dental assistants who } \\
\text { used inhalant anesthetics } \\
\text { ( } \mathrm{N}=3724) \\
\text { Unexposed: dentists and } \\
\text { dental assistants not using } \\
\text { inhalant anesthetics } \\
(\mathrm{N}=8893)\end{array}$ & $1968-1977$ & $\begin{array}{l}\text { Postal } \\
\text { question- } \\
\text { naire }\end{array}$ & $\begin{array}{l}\text { One year } \\
\text { prior to } \\
\text { conception } \\
\text { trimester }\end{array}$ & $\begin{array}{l}\text { Maternall } \\
\text { paternal }\end{array}$ & $\begin{array}{l}\text { Up to } 10 \\
\text { years of } \\
\text { age }\end{array}$ & $\begin{array}{l}5.4 \% \text { malformations } \\
\text { among female dental as- } \\
\text { sistant users vs } 3.6 \% \text { in } \\
\text { nonusers ( } P=0.02 \text {, espe- } \\
\text { cially MS for using ni- } \\
\text { trous oxide); no dose- } \\
\text { response; no difference } \\
\text { in offspring of male den- } \\
\text { tists }\end{array}$ \\
\hline \multicolumn{8}{|c|}{ Agricultural chemicals } \\
\hline $\begin{array}{l}\text { Schwartz et al } \\
\text { (72) }\end{array}$ & $\begin{array}{l}\text { All births in California } \\
\text { agricultural area }(\mathrm{N}=2463 \\
\text { singleton births) } \\
\text { Exposed: parents with } \\
\text { agricultural jobs }(\mathrm{N}=990) \\
\text { Unexposed: parents in } \\
\text { nonagricultural jobs } \\
\text { ( } \mathrm{N}=1365)\end{array}$ & $1975-1978$ & $\begin{array}{l}\text { Mothers' } \\
\text { \& infants' } \\
\text { records }\end{array}$ & $\begin{array}{l}\text { During } \\
\text { pregnancy }\end{array}$ & $\begin{array}{l}\text { Maternal/ } \\
\text { paternal }\end{array}$ & $\begin{array}{l}\text { Not } \\
\text { stated }\end{array}$ & $\begin{array}{l}\mathrm{RR}=2.3 \text { for limb reduc- } \\
\text { tion defects }(5.07 / 1000 \text { in } \\
\text { agricultural vs } 2.22 / 1000 \\
\text { in other jobs, total limb } \\
\text { defects }=8) \text {; } \\
\text { no other differences }\end{array}$ \\
\hline $\begin{array}{l}\text { Smith et al }(77) \\
\text { and Smith } \\
\text { et al (78) }\end{array}$ & $\begin{array}{l}\text { Exposed: New Zealand } \\
\text { chemical applicators in } \\
\text { agricultural chemical board } \\
(N=459) \\
\text { Unexposed: agricultural } \\
\text { contractors }(N=422)\end{array}$ & $1969-1979$ & $\begin{array}{l}\text { Postal } \\
\text { question- } \\
\text { naire }\end{array}$ & $\begin{array}{l}\text { One year } \\
\text { prior to } \\
\text { conception } \\
\text { and during } \\
\text { pregnancy }\end{array}$ & Paternal & $\begin{array}{l}\text { Not } \\
\text { stated }\end{array}$ & $\begin{array}{l}2 \% \text { malformations } \\
\text { among exposed; } 1.6 \% \\
\text { among unexposed }\end{array}$ \\
\hline Roan et al (67) & $\begin{array}{l}\text { Exposed: offspring of } \\
\text { American NAAA members } \\
\text { ( } N=588 \text { live births) } \\
\text { Unexposed: offspring of } \\
\text { sibling of NAAA member } \\
(\mathrm{N}=350 \text { live births })\end{array}$ & $\begin{array}{l}\text { Not } \\
\text { stated }\end{array}$ & $\begin{array}{l}\text { Postal } \\
\text { question- } \\
\text { naire }\end{array}$ & $\begin{array}{l}\text { Not } \\
\text { stated }\end{array}$ & Paternal & Childhood & $\begin{array}{l}5.9 \% \text { maiformations } \\
\text { among exposed; } 6.4 \% \\
\text { among unexposed }\end{array}$ \\
\hline $\begin{array}{l}\text { Townsend } \\
\text { et al (85) }\end{array}$ & $\begin{array}{l}\text { Exposed: at least one } \\
\text { month in chlorophenol } \\
\text { process in Michigan plant } \\
(\mathrm{N}=637 \text { live births) } \\
\text { Unexposed: employees at } \\
\text { least one month not in } \\
\text { potential exposed area, } \\
\text { matched by date of hire } \\
(\mathrm{N}=1785 \text { live births) }\end{array}$ & $1939-1975$ & $\begin{array}{l}\text { Company } \\
\text { records and } \\
\text { question- } \\
\text { naire }\end{array}$ & $\begin{array}{l}\text { Any time } \\
\text { prior to } \\
\text { conception }\end{array}$ & Paternal & $\begin{array}{l}\text { One year } \\
\text { after } \\
\text { birth }\end{array}$ & $\begin{array}{l}\text { No difference in malfor- } \\
\text { mations among dioxin or } \\
\text { TCDD exposed }\end{array}$ \\
\hline $\begin{array}{l}\text { Erickson et al } \\
\text { (21) }\end{array}$ & $\begin{array}{l}\text { Cases: serious structural } \\
\text { malformations in MACDP } \\
(N=4929) \\
\text { Referents: liveborn from } \\
\text { vital records }(N=3029)\end{array}$ & $1968-1980$ & Interviews & $\begin{array}{l}\text { Prior to } \\
\text { conception }\end{array}$ & Paternal & $\begin{array}{l}\text { One year } \\
\text { after } \\
\text { birth }\end{array}$ & $\begin{array}{l}\text { Suggestion of dose- } \\
\text { response for Agent } \\
\text { Orange exposed persons } \\
\text { and spina bifida and cleft } \\
\text { lip with or without palate; } \\
\text { excess of second affected } \\
\text { child in Agent Orange } \\
\text { group; no excess risk } \\
\text { overall for Agent Orange; } \\
\text { no excess risk for } \\
\text { Vietnam veterans }\end{array}$ \\
\hline
\end{tabular}


Table 1. Continued.

\begin{tabular}{|c|c|c|c|c|c|c|c|}
\hline Reference & Subjects & $\begin{array}{l}\text { Study } \\
\text { period }\end{array}$ & $\begin{array}{l}\text { Source of } \\
\text { occupational } \\
\text { information }\end{array}$ & $\begin{array}{l}\text { Timing of } \\
\text { exposure }\end{array}$ & $\begin{array}{l}\text { Maternall } \\
\text { paternal } \\
\text { exposure }\end{array}$ & $\begin{array}{l}\text { Length of } \\
\text { ascertain- } \\
\text { ment of mal- } \\
\text { formations }\end{array}$ & Results \\
\hline \multicolumn{8}{|c|}{ Industrial chemicals } \\
\hline $\begin{array}{l}\text { Nordstrom } \\
\text { et al }(59)\end{array}$ & $\begin{array}{l}\text { Exposed: worker in Swedish } \\
\text { smeltery during pregnancy } \\
\text { Unexposed: no work in } \\
\text { smeltery during pregnancy } \\
(\mathrm{N}=\text { not stated) }\end{array}$ & $1955-1976$ & $\begin{array}{l}\text { Question- } \\
\text { naires and } \\
\text { personnel } \\
\text { records }\end{array}$ & $\begin{array}{l}\text { During } \\
\text { pregnancy }\end{array}$ & Maternal & $\begin{array}{l}\text { Not } \\
\text { stated }\end{array}$ & $\begin{array}{l}5.8 \% \text { malformations in } \\
\text { exposed and } 2.2 \% \text { in } \\
\text { unexposed }(P<0.005) \text {; } \\
\text { increase over time }\end{array}$ \\
\hline $\begin{array}{l}\text { Blomquist et al } \\
\text { (7) }\end{array}$ & $\begin{array}{l}\text { Exposed: pregnancy in } \\
\text { women working in Swedish } \\
\text { pulp and paper industry } \\
\text { ( } \mathrm{N}=890) \\
\text { Compared to total popula- } \\
\text { tion expected prevalence }\end{array}$ & $1973-1977$ & $\begin{array}{l}\text { Postal } \\
\text { question- } \\
\text { naires to } \\
\text { industry }\end{array}$ & $\begin{array}{l}\text { During } \\
\text { pregnancy }\end{array}$ & Maternal & $\begin{array}{l}\text { One week } \\
\text { of life }\end{array}$ & $\begin{array}{l}\text { Twofold excess of malfor- } \\
\text { mations in offspring of } \\
\text { women working in labora- } \\
\text { tories or in the refining of } \\
\text { paper }\end{array}$ \\
\hline $\begin{array}{l}\text { Edmonds et al } \\
(18)\end{array}$ & $\begin{array}{l}\text { Cases: verified CNS defects } \\
\text { in Ohio BDMP or vital } \\
\text { statistics }(N=41) \\
\text { Referents: live born from } \\
\text { birth certificate matched on } \\
\text { birth month, race, paternal } \\
\text { education and maternal age } \\
(\mathrm{N}=41)\end{array}$ & $1970-1974$ & $\begin{array}{l}\text { Telephone } \\
\text { interview }\end{array}$ & $\begin{array}{l}\text { Five years } \\
\text { prior to con- } \\
\text { ception and } \\
\text { during } \\
\text { pregnancy }\end{array}$ & $\begin{array}{l}\text { Maternall } \\
\text { paternal }\end{array}$ & At birth & $\begin{array}{l}\text { No difference in paternal } \\
\text { employment in PVC plant } \\
\text { or in distance from plant } \\
\text { at conception; no differ- } \\
\text { ence in maternal employ- } \\
\text { ment in PVC plant }\end{array}$ \\
\hline $\begin{array}{l}\text { Axelson et al } \\
\text { (1) }\end{array}$ & $\begin{array}{l}\text { Exposed: women working in } \\
\text { Swedish tirebuilding during } \\
\text { pregnancy ( } \mathrm{N}=21 \text { pregnan- } \\
\text { cies) } \\
\text { Unexposed: women not } \\
\text { working in tirebuilding dur- } \\
\text { ing pregnancy ( } \mathrm{N}=63 \text { preg- } \\
\text { nancies) }\end{array}$ & $1958-1980$ & Interview & $\begin{array}{l}\text { Not } \\
\text { stated }\end{array}$ & Maternal & $\begin{array}{l}\text { Not } \\
\text { stated }\end{array}$ & $\begin{array}{l}\mathrm{RR}=1.0 \text { for exposed vs } \\
\text { unexposed malformation } \\
\text { groups; four times more } \\
\text { abortions and malforma- } \\
\text { tions combined in ex- } \\
\text { posed group }\end{array}$ \\
\hline $\begin{array}{l}\text { Lindbohm et al } \\
(50)\end{array}$ & $\begin{array}{l}\text { Cases: all malformations, } \\
\text { Finnish Register ( } \mathrm{N}=48) \\
\text { Referents: two normal } \\
\text { births per case, matched on } \\
\text { delivery date and chapter } \\
\text { Exposed: joined union no } \\
\text { later than two and a half } \\
\text { months after conception or } \\
\text { did not resign before sixth } \\
\text { week of pregnancy } \\
\text { Unexposed: union members } \\
\text { in factory not meeting ex- } \\
\text { posure criteria }\end{array}$ & $1973-1977$ & $\begin{array}{l}\text { Registry and } \\
\text { union } \\
\text { records }\end{array}$ & $\begin{array}{l}\text { First } \\
\text { trimester }\end{array}$ & Maternal & One year & $\begin{array}{l}\text { No increased risk for mal- } \\
\text { formation in any employ- } \\
\text { ment category of rubber } \\
\text { and leather workers }\end{array}$ \\
\hline
\end{tabular}

\section{Miscellaneous}

Kallen et al

(41)

\section{Exposed: Swedish female physiotherapists $(\mathrm{N}=2018$ \\ pregnancies)}

Unexposed: all others in

birth register; nested case-

referent of malformations or

death $(\mathrm{N}=36)$

Referents matched on

maternal age, parity, birth

date, $(N=74)$

Logue et al Nested study of American (52)

male physiotherapists

Cases: all malformation

$(\mathrm{N}=169)$

Referents: matched on year

of birth and maternal age

$(\mathrm{N}=338)$

Kurppa et al Cases: all malformations in (48) Finland $(N=1475$ )

Referents: mother immediately preceding case matched on time and area of birth $(N=1475)$

McDonald et al Exposed: current and previ(55)

\begin{tabular}{|c|c|c|c|c|c|}
\hline $1973-1978$ & $\begin{array}{l}\text { Records and } \\
\text { postal } \\
\text { question- } \\
\text { naire }\end{array}$ & $\begin{array}{l}\text { At birth } \\
\text { and during } \\
\text { pregnancy }\end{array}$ & Maternal & Birth & $\begin{array}{l}\text { More frequent use of } \\
\text { shortwaves and ultra- } \\
\text { sound among cases } \\
\text { ( } P=0.03 \text { and } P<0.10 \text {, } \\
\text { respectively); no pattern } \\
\text { of malformations }\end{array}$ \\
\hline
\end{tabular}

\begin{tabular}{|c|c|c|c|c|c|}
\hline $\begin{array}{l}\text { Not } \\
\text { stated }\end{array}$ & $\begin{array}{l}\text { Postal } \\
\text { question- } \\
\text { naire }\end{array}$ & $\begin{array}{l}\text { Any time } \\
\text { prior to con- } \\
\text { ception and } \\
\text { three months }\end{array}$ & Paternal & $\begin{array}{l}\text { Not } \\
\text { stated }\end{array}$ & $\begin{array}{l}\text { No difference between } \\
\text { cases and referents for } \\
\text { preconceptional exposure } \\
\text { to nonionizing radiation }\end{array}$ \\
\hline
\end{tabular}

1976-1982 Interview First Maternal One year

No increased risk for VDT exposure; 51 case vs 60 reference mothers exposed $(\mathrm{OR}=0.9$,

$95 \% \mathrm{Cl}=0.6-1.2$ )

1982-1984 Interview

When women Maternal became aware of pregnancy

At birth for current pregnancies; not stated for previous pregnancies
Malformation in $3.3 \%$ of $3.7 \%$ in nonusers for current pregnancies; $3.8 \%$ vs $3.6 \%$ for previous pregnancies offspring of VDT users; 
Table 1. Continued.

\begin{tabular}{|c|c|c|c|c|c|c|c|}
\hline Reference & Subjects & $\begin{array}{l}\text { Study } \\
\text { period }\end{array}$ & $\begin{array}{l}\text { Source of } \\
\text { occupational } \\
\text { information }\end{array}$ & $\begin{array}{l}\text { Timing of } \\
\text { exposure }\end{array}$ & $\begin{array}{l}\text { Maternal/ } \\
\text { paternal } \\
\text { exposure }\end{array}$ & $\begin{array}{l}\text { Length of } \\
\text { ascertain- } \\
\text { ment of mal- } \\
\text { formations }\end{array}$ & Results \\
\hline $\begin{array}{l}\text { Bjerkedal }(5) \\
\text { and Bjerkedal } \\
(6)\end{array}$ & $\begin{array}{l}\text { First-borns in Norway } \\
(\mathrm{N}=103794) ; \\
3213 \text { with malformations, } \\
\text { all types }\end{array}$ & $1970-1973$ & $\begin{array}{l}1970 \text { census } \\
\text { records }\end{array}$ & $\begin{array}{l}\text { Variable, up } \\
\text { to } 38 \text { months } \\
\text { or during } \\
\text { pregnancy }\end{array}$ & Maternal & $\begin{array}{l}\text { First year } \\
\text { of life }\end{array}$ & $\begin{array}{l}\text { Crude } R R=1.12(P<0.01) \\
\text { for women economically } \\
\text { active during pregnancy; } \\
\text { significant RR for occu- } \\
\text { pations in textile and } \\
\text { leather (RR }=0.77) \text {, cleri- } \\
\text { cal }(R R=1.19 \text { ), restaurant, } \\
\text { hotel, domestic } \\
\text { (RR }=1.15) \text {, technical } \\
\text { (RR }=1.46) \text {, and nursing } \\
\text { (RR }=1.32 \text { ) work }\end{array}$ \\
\hline $\begin{array}{l}\text { Erickson et al } \\
\text { (20) }\end{array}$ & $\begin{array}{l}\text { Metropolitan Atlanta BDMP } \\
\text { - compare each malforma- } \\
\text { tion to all other malforma- } \\
\text { tions and to occupations in } \\
\text { Atlanta at the census } \\
(\mathrm{N}=989)\end{array}$ & $\begin{array}{l}\text { Not } \\
\text { stated }\end{array}$ & $\begin{array}{l}\text { Interview } \\
\text { with } \\
\text { mothers }\end{array}$ & $\begin{array}{l}\text { Maternal, } \\
\text { two years } \\
\text { prior to con- } \\
\text { ception until } \\
\text { birth; pater- } \\
\text { nal, at con- } \\
\text { ception }\end{array}$ & $\begin{array}{l}\text { Maternal/ } \\
\text { paternal }\end{array}$ & $\begin{array}{l}\text { First year } \\
\text { of life }\end{array}$ & $\begin{array}{l}\text { Significant risks in first } \\
\text { trimester: OR }=3.3 \text { for } \\
\text { CNS and maternal sales } \\
\text { worker; } O R=2.3 \text { for CLP } \\
\text { and maternal health } \\
\text { worker; OR }=21.2 \text { for CLP } \\
\text { and maternal clerical } \\
\text { worker; OR }=7.1 \text { for CLP } \\
\text { and maternal business } \\
\text { and repair worker; } \\
\text { OR = 5.1 for reduction } \\
\text { deformity and maternal } \\
\text { service retail worker. Sig- } \\
\text { nificant risk preconcep- } \\
\text { tion: OR }=38.6 \text { for gas- } \\
\text { troschisis and om- } \\
\text { phalocele and maternal } \\
\text { other crafts; many pater- } \\
\text { nal exposures significant }\end{array}$ \\
\hline $\begin{array}{l}\text { Hemminki (29) } \\
\text { and Hemminki } \\
\text { et al (31) }\end{array}$ & $\begin{array}{l}\text { Cases: CNS, oral cleft and } \\
\text { MS malformations - Finn- } \\
\text { ish Register }(\mathrm{N}=1650) \\
\text { Referents: previous birth, } \\
\text { matched on time and place } \\
\text { of birth }\end{array}$ & $1967-1977$ & $\begin{array}{l}\text { Interview } \\
\text { during } \\
\text { pregnancy }\end{array}$ & $\begin{array}{l}\text { During } \\
\text { pregnancy, } \\
\text { usually } \\
\text { third month }\end{array}$ & $\begin{array}{l}\text { Maternal/ } \\
\text { paternal }\end{array}$ & $\begin{array}{l}\text { First year } \\
\text { of life }\end{array}$ & $\begin{array}{l}\text { CNS }(P<0.05): O R=1.57 \\
\text { maternal industry and } \\
\text { construction worker, } \\
\text { OR }=2.0 \text { maternal } \\
\text { academic degree; } \\
\text { clefts }(P<0.05) \text { : OR }=1.94 \\
\text { maternal transportation or } \\
\text { communication worker, } \\
\text { OR }=3.1 \text { maternal phone } \\
\text { operators, OR }=2.5 \text { mater- } \\
\text { nal teacher; } M S(P<0.05) \text { : } \\
\text { OR }=1.3 \text { maternal indus- } \\
\text { trial worker OR }=1.8 \\
\text { maternal food industry } \\
\text { worker, OR }=1.7 \text { maternal } \\
\text { factory worker, OR }=5.0 \\
\text { maternal gardener, } \\
\text { OR }=1.7 \text { maternal not } \\
\text { specified; no increase } \\
\text { with paternal exposures }\end{array}$ \\
\hline Papier (62) & $\begin{array}{l}\text { All malformations in one } \\
\text { Israel hospital ( } N=1481 \\
\text { malformations, } 35542 \\
\text { births) }\end{array}$ & $1966-1976$ & $\begin{array}{l}\text { Question- } \\
\text { naires } \\
\text { prenatally }\end{array}$ & $\begin{array}{l}\text { Not } \\
\text { stated }\end{array}$ & $\begin{array}{l}\text { Maternalf } \\
\text { paternal }\end{array}$ & $\begin{array}{l}\text { First } 4 d \\
\text { of life }\end{array}$ & $\begin{array}{l}\text { No maternal or paternal } \\
\text { occupational category } \\
\text { showed an excess }\end{array}$ \\
\hline $\begin{array}{l}\text { Polednak \& } \\
\text { Janerich (66) }\end{array}$ & $\begin{array}{l}\text { Cases: anencephaly or } \\
\text { spina bifida - New York } \\
(N=171) \\
\text { Referents: adjacent birth, } \\
\text { matched on maternal age } \\
\text { and race }(N=171)\end{array}$ & $1968-1974$ & $\begin{array}{l}\text { Birth } \\
\text { records }\end{array}$ & Birth & $\begin{array}{l}\text { Maternal/ } \\
\text { paternal }\end{array}$ & $\begin{array}{l}\text { At } \\
\text { birth }\end{array}$ & $\begin{array}{l}\text { No maternal exposure } \\
\text { showed an excess; } \\
\text { increased risk with paternal } \\
\text { farmer, auto mechanic or } \\
\text { electrical engineer or } \\
\text { technician }\end{array}$ \\
\hline
\end{tabular}

malformations to study are derived from findings in prior epidemiologic investigations, astute clinical observations, or animal teratogenic experiments.

Data derived from animal experiments have shown that a number of chemical substances can interfere with different phases of the reproductive cycle and are teratogenic in animal species $(17,75)$. However, another generic problem for studies of malformations is that, even for substances that have been described as teratogenic, controversy exists with respect to extrapolation from animal data to human experience. Such extrapolation is hindered by differences in metabolism, placental function, and patterns of development among animal species $(68,81)$. Thus the critical tim- ing of exposure to particular teratogens may not be generalizable across species (81). Furthermore, Saxen (68) has noted that, for some substances found to be teratogenic in humans, an effect has not been found in experimental studies.

\section{Prevalence estimation}

The prevalences of congenital malformations can be influenced by variations in the source of information from which malformations are ascertained, the length of time subsequent to birth during which malformations are detected, and the diagnostic definition of a specific malformation. There is potential for biased 
study results if such variations are differentially exerted in exposed and unexposed groups. While these issues are generic to most epidemiologic studies of malformations, they must be considered specifically for investigations of parental occupational exposures and malformations in offspring.

The ascertainment of children with malformations has been accomplished from a variety of information sources, including vital statistics records such as birth, death and fetal death certificates, interviews with parents, medical records, and both active and passive reporting or surveillance systems (table 1). The use of different ascertainment sources may result in varying prevalence estimates for a given malformation in that greater care may be taken to ensure complete and accurate information from some sources of information than from others. For example, while birth certificates are frequently used to ascertain malformations (table 1 , references 60 and 66 ) because of the relative ease and low cost, this method is well-known to underestimate the prevalence of malformations $(4,10,25)$ when compared to, say, a surveillance system such as the California Birth Defects Monitoring Program (26) or the Metropolitan Atlanta Congenital Defects Program (19), which actively monitor for malformations up to one year of life. However, some of the underreporting associated with the use of birth certificates is merely due to failure to record malformations on the birth certificate (69). Reporting has also been found to vary by size of the hospital of birth (4). It has been suggested that data on malformations obtained from interviews with parents, particularly in prospective studies (a method of ascertainment for many of the studies in table 1) be considered with caution (3).

An additional concern generic to studies of malformations is that the length of time into the postnatal period when a malformation is diagnosed varies considerably among studies (table 1). Duration of postnatal follow-up needs to be considered because both the prevalence and types of congenital malformations diagnosed change with time subsequent to birth. For example, anencephaly is generally noted at time of delivery, but many of the cardiac malformations may not be diagnosed until the first six months of life.

Another concern pertains to how a particular malformation is defined. Reporting varies by the nature and severity of the malformation $(4,10,25,46)$. Although less of an issue with severe malformations because they are usually more easily diagnosed, if the diagnostic criteria differ by reporting source or between studies for a particular malformation, so will the prevalence estimates. As an example, if the case definition for a particular cardiac lesion in one study specifies that the confirmed diagnosis be based on an invasive diagnostic procedure, whereas in another study such stringent criteria are not part of the case definition, then different prevalence estimates may be observed. A further problem associated with the definition of congenital malformations is whether to include stillbirths (37). The prevalence of malformations among stillborn infants is higher than among infants born live (87). Thus including or excluding stillbirths will affect the magnitude of the reported prevalence.

In addition to the potential variability in prevalence estimates due to differences in ascertainment source, length of follow-up, and inconsistent case definitions, malformations may be differentially reported in certain study subgroups, such as certain ethnic, educational, or economic groups $(38,53)$, that have differing access to medical care for the diagnosis of malformations. For example, for workers' families covered by a health plan there may be better ascertainment than for workers' families without such coverage. It has further been observed that the reporting of some congenital malformations is greater in rural than urban areas (54).

\section{Estimation of occupational exposures}

\section{Biological considerations of occupational exposures}

Specifying hypotheses with a firm biological rationale helps reduce both false positive and false negative results. Maternal or paternal exposures to putative agents preconceptionally or postconceptionally may be related to the birth of a malformed child $(39,42)$. Crucial to this understanding is the specific timing of such exposures in the reproductive cycle, a factor critical for the assessment of an agent's teratogenic risk (17).

Preconceptional occupational exposures of either parent could theoretically contribute to the subsequent development of offspring with a malformation. Exposures in the workplace may cause chromosome damage or changes in molecular deoxyribonucleic acid among parental germ cells, men being somewhat more likely to be affected since there are numerous opportunities when agents can have an impact during cell divisions in sperm production (82). However, Pearn (63) noted that there is, to date, no experimental evidence to suggest that paternal exposure to agents can result in a teratogenic effect. Neither paternal nor maternal preconceptional occupational exposures have been extensively examined with regard to the risk of malformations in offspring $(5,6,14,18,20,21,52$, $77,78,85)$. On the other hand, much more is known about how postconceptional occupational exposures may affect normal embryologic development $(2,7,16$, $22-24,29,30,34-36,45,47,48,50,55,59,72$ ).

The critical period of teratogenesis is thought to coincide the best with the period of embryonic organogenesis, from the third to the twelfth week of gestation $(58,86)$. Within this period, there is a critical period for each organ system during which dysmorphogenesis might occur. The exact time of vulnerability during the critical periods varies with the agent, as well as with dose and duration of exposure $(58,86)$. The 
application of the critical period concept may be epidemiologically useful in identifying potential occupational teratogens. If exposure to an agent occurs prior to the end of a given critical gestational period, it can be considered a possible teratogen. However, occupational exposure of a pregnant woman after closure of the fetal palate cannot, for example, be a cause of cleft palate.

Defining exposure time relative to embryologic development allows for a greater likelihood of identifying a particular agent or agents as possible teratogens, ie, random misclassification of exposure is less likely, and therefore the estimated effect is less likely to be underestimated. However, imprecision may exist in the estimation of fetal age, often because the actual date of conception is not known but is, rather, estimated according to the date of last menses derived from medical records or estimated by the recollection of the person in question. The latter estimation is susceptible to variations in a woman's self-awareness of the beginning of her pregnancy, eg, a mother may perceive her pregnancy to have begun when she felt pregnant, possibly several weeks subsequent to when her pregnancy actually began. Such imprecision may lead to variations in the estimation of critical periods (58) and the misclassification of exposure status. Unless the proportion of truly exposed among those classified as exposed is relatively large or the effect of the exposure on the particular studied outcome is large (80), the effect could go undetected. To minimize this type of misclassification, study instruments must be constructed to elicit more precise information about the time of conception.

An additional issue of biological concern is that of the exposure-response relationship. In most studies of occupational exposures related to congenital malformations, it is difficult to assess the extent of exposure accurately. Thus surrogates of actual exposure are often used, such as duration of exposure. As a result, there is little information in the literature about exposure in humans, and it remains unclear if high exposures for short or intermittent periods or low exposures for longer periods are equivalent in risk even within the limits of the relevant embryologic timing of exposure. Other models of risk of adverse reproductive outcomes associated with exposure have been proposed (73).

\section{Methodological considerations for occupational exposures}

The validity of reported occupational exposures is crucial to risk estimation and depends on the following two related areas of concern: the source of the exposure information and the inclusion of validity checks on this information. As can be seen in table 1, the most frequent sources of information about occupational exposures in studies of congenital malformations are birth certificates, interviews with or questionnaires for one or both parents, and, occasionally, company records. Each of these techniques has its advantages and disadvantages as a source of occupational exposure data.

Source of information. The major advantages of the birth certificate as a source are ease of access and reduced cost. However, the many disadvantages of this source tend to outweigh its advantages. Concern about using birth certificates to acquire occupational exposure data results from the following possibilities. The data may be incomplete since, in many areas, ascertainment of parental occupation is not mandatory for the birth certificate (82). In addition, the recorded parental occupation at the time of birth on the birth certificate may not be the parent's usual occupation or occupation at conception or at the relevant time during pregnancy. As an example, the mother's occupation often appears as housewife at the time of birth when in fact she may have worked in another capacity at the time of conception or during the early months of her pregnancy (11). Errors in classifying occupational titles into exposed or unexposed categories may also bias the effect measure since specific occupational exposures are not recorded on the birth certificate and occupational title must be used as a surrogate measure. Occupational titles used as a surrogate may be too crude for exposure estimation (35). Several occupationexposure linkage strategies have been proposed in which occupations or occupation-industry combinations are linked with specific exposures $(13,33,57,76)$. Two indirect strategies $(33,57)$, however, have been shown to have poor agreement with direct reporting based on questionnaire-elicited data (51). Moreover, information on occupation may not be collected or coded in a standardized way, and thus it may be noncomparable and somewhat inappropriate for purposes of aggregating these data from various areas or hospitals. Finally, the information obtained from the birth certificate about potential confounders may be limited.

The primary advantages of occupational data obtained from interviews and questionnaires are that subjects can be queried directly, in a detailed and standardized way, about occupations and occupational exposures, including information on substances, timing, exposures, protective gear, and work habits. There are, however, a number of disadvantages with this approach. First, while respondents know about terms of employment and character of the job (64), they may not know with certainty about exposures they have had on the job, many of which change over the time of employment and thus introduce the possibility of misclassification. Second, one parent may be easier to find and more accessible and may thus become the respondent. Yet, it is often unclear if maternal or paternal occupational exposures, or both, are the most relevant, and the one parent may not provide valid and complete information regarding the other's specific occupa- 
tional exposures and is more likely to know a specific job title than specific workplace exposures (74).

Finally, this technique is probably the most susceptible to problems of information bias (70). The underlying reason for concern about this bias is that parents of children with malformations may recall exposures better than parents of nonaffected children, the result being a biased estimate of the effect measure away from the null. Thus the likelihood increases for incorrectly claiming that a difference in exposure exists when it really does not. However, a number of studies examining exposures during pregnancy and specifically testing for recall bias have been unable to demonstrate its presence $(43,61,84,88)$. Several suggestions have appeared in the literature for minimizing recall bias. First, asking specific questions about exposures (eg, in the case of drugs, the conditions for which taken, and the names) tends to improve recall, whereas open-ended questions do not enhance information (56). With regard to occupational exposures, asking about specific exposures rather than trying to deduce exposure from job categories may yield more information (51).

The primary advantages of using company records are that they are not subject to problems of recall bias and tend to be more complete in recording occupational exposures. There are, however, some problems with this technique as well. Perhaps the two most looming potential problems are that of gaining access to company personnel records and that of the lack of recording changes in exposures over time. Since companies are not universally cooperative in sharing their personnel records for studies of potential adverse health effects of occupational exposures, lack of cooperation can compromise the completeness and validity of data obtained in this way. Using company records to determine occupational exposures is also a rather cumbersome technique to be used in a case-referent study, since it often involves obtaining cooperation from many different companies. In addition, exposures for any given job title or position may change over time, but exposure changes may not be routinely recorded in the personnel file. Thus company records might be of less value, in some instances, in obtaining valid exposure information than interviews with the workers themselves. Furthermore, information on potential confounders from company records may be insufficient.

Validating exposure information. Because no single technique for obtaining occupational exposure information is perfect, the need for building validity checks into studies of such exposures is critical. Experienced occupational hygienists can certainly contribute to the process of validating occupational exposure information. Validating not only positive reports of exposure, but negative ones as well, would minimize the chances of misclassification bias. Sometimes it may only be necessary to perform validity checks on a sample of the total population to determine that the selected method for obtaining data on occupational exposures is sufficiently valid and complete, while at other times it may be necessary to validate information on all subjects. There are, however, problems in attempting to validate information. First, sources for validation are not always available. Second, it is not always clear what information source should be considered the valid measure of exposure if two sources disagree. For example, medical records are often considered the valid source of information in epidemiologic studies, but some investigators have found that these records may be as incomplete or inaccurate as other sources of data (32). Finally, more recently the use of biological markers in epidemiologic research has been increasing. Although the recent study conducted by the Centers for Disease Control (12) involving serum levels of dioxin serves as an example of how useful biological measures may be for validating another source of exposure information, the validity and meaning of many biological markers will still need to be established before used in this regard (71).

\section{Other design and analytical considerations}

\section{Reference groups}

Inclusion of a reference group which will provide an unbiased estimate of risk requires careful consideration of likely modes, mechanisms, and timing of exposures, ie, an estimate not biased due to differential error in the ascertainment of exposure status. In prospective studies of occupational exposures in general, and specifically those studying these exposures as related to the occurrence of congenital malformations, a frequent approach is to include as a reference group another group of workers in the same industry which is presumed not to have had the exposure under study. It is usually easier to obtain permission and cooperation for such a group because they have already been obtained for the exposed group. The ascertainment and the follow-up of subjects may also be easier. In addition, this method makes it more likely that the reference group will experience medical surveillance similar to that of the exposed group than a reference group selected from outside the industry.

Problems generic to occupational studies using a reference group from the same industry include (i) the possibility of the workers changing positions within the same industry, the result being changed exposure status over time, and (ii) the possibility of the reference or unexposed group being inadvertently exposed to the substance of interest. These types of problems result in a misclassification of exposure that biases risk estimates toward the null. One must determine whether the advantages of using this type of reference group outweigh the disadvantage of possibly providing false reassurance about a potential hazard. 
The choice of an appropriate reference group in a case-referent study may also be problematic. The most frequently used reference groups are infants with malformations other than the one under study or normal infants (eg, siblings, neighbors, friends, a probability sample of all infants in the area under study, or random-digit-dialed referents). Using infants with other malformations helps to control recall bias because parents with affected infants may try harder to recall exposures which may have affected their offspring adversely. Another advantage of a reference group with other malformations is that cooperation may be better and the subjects may be easier to follow-up than normal infants, the result being a reduction in the possibility for response bias.

One drawback to consider in using a reference group comprised of infants with malformations is that, since the etiology of about $65 \%$ of all congenital malformations is unknown (87), some of the reference group malformations may be etiologically linked with the exposure under study. Because much of organogenesis occurs in the first trimester, it is likely that more than one organ system could be adversely affected by the same exposure during that 12 -week period. One alternative is to use a reference group with a malformation whose etiology is known, eg, a chromosome malformation, although this may not be as good a control for recall bias if the etiology is well-known to the parents. One could also include a variety of diagnostic groups of malformations to minimize the impact of inadvertently including one that is associated with the exposure under study $(40,70)$. However, because the etiology of most malformations is unknown, the likelihood of including more than one malformation that is associated with the exposure under study may still be great and may thus result in an underestimate of the effect measure. Another possibility is to use a nondiseased reference group which would provide the opportunity to compare exposure frequency to that of a group that has not been influenced by disease but would enhance the possibility of recall bias.

An additional option would be to use two reference groups, one with other malformations and one without. This method would permit both recall bias and risk to be assessed (70). It, however, is more difficult and costly and could either complicate or elucidate $(15,28)$ the interpretation of results if effect measures differ substantially for the different comparison groups or if the explanation of the discrepancy can be deduced.

One further issue regarding the selection of a reference group is which subjects should be excluded. Some questions that arise in considering exclusions are (i) whether referents should be excluded who have malformations which may be associated with the exposure under study, ie, how strong must the association be for the referent to be excluded; (ii) what should be done with referents for whom the association with exposure is speculative (perhaps they should be included as a separate group for analysis); and (iii) what referents should be excluded when the study is exploratory (ie, a number of exposures may be studied which may be associated with different malformations in the reference group).

\section{Sample size and multiple tests}

The issue of sufficient study power for examining specific malformations needs to be considered. Because specific malformations are rare, the practice of broadly grouping malformations is often undertaken and justified on the grounds of increasing sample size with concomitant increases in study power. However, one is hardpressed on biological grounds to support a practice that, even though it may result in the ability to detect a smaller effect measure as statistically significant, may well obscure interpretation of a potential specific exposure-malformation relation. However, grouping specific malformations may have biological validity if several malformations suspected to have a similar etiology or embryology are considered as a single group or if complex mixtures of exposures, eg, in toxic waste sites, are of interest or if exposure occurs when a number of organ systems are developing. A number of the prospective studies reviewed in table 1 considered congenital malformations as a single end point $(1,23,27,49)$.

Inherent in a prospective examination of congenital malformations in which the frequency of numerous malformations is examined between exposed and unexposed groups is not only the problem of small numbers and reduced power, but also the problem of interpreting findings from multiple testing. There are approximately 200 specific congenital malformations at the four-digit level of the International Classification of Diseases (ninth revision). If relative risks are calculated for each outcome, one would expect 10 to be statistically significant by chance alone at an alpha level of 0.05 . Frequently, multiple exposures and multiple time periods are also under consideration, a process which further increases the number of statistical tests and potential false positives. Certainly, if several indicators of the same exposure are available, one can examine the consistency of the effect estimates across the various indicators. However, if more than one estimate of the same exposure is not available, one is then left with the difficult choice of which positive associations to pursue. Several approaches might be considered for reporting results when a large number of potential associations have been examined, including the reporting of all potential associations examined with the thought that each exposure-outcome relation is a separate investigation (8) or the reporting of only associations which are "significant" after an adjustment procedure such as the Bonferroni (79) procedure or the reporting of all potential associations examined but providing a ranking in order of priority for investigations using empirical Bayes techniques (83). 
Potentially hazardous workplace exposures have raised concern regarding the adverse reproductive effects of such exposures to men and women. Epidemiologic studies of these relations are important for assessing such risks so as to help in setting standards to reduce hazardous exposures and the risk of malformations. Addressing the methodologic concerns discussed in this review regarding exposure and outcome in future studies of occupational exposures and congenital malformations will likely result in greater specificity of findings and thus enhance the possibilities for prevention.

\section{Acknowledgments}

The authors gratefully acknowledge the assistance of Ms L Croen, Drs L Gordis, J Harris, and C Torfs and Ms M Torres in the preparation of this manuscript.

\section{References}

1. Axelson O, Edling C, Anderson L. Pregnancy outcome among women in a Swedish rubber plant. Scand J Work Environ Health 9 (1983) 79-83.

2. Axelsson G, Lutz C, Rylander R. Exposure to solvents and outcome of pregnancy in university laboratory employees. Br J Ind Med 41 (1984) 305-312.

3. Axelsson G, Rylander R. Validation of questionnaire reported miscarriage, malformation and birth weight. Int J Epidemiol 13 (1984) 94-98.

4. Bakewell JM, Land G. Completeness of congenital anomalies reported on the Missouri birth certificate: Comparison with the hospital discharge abstract. Missouri Department of Social Services, Jefferson City, MI 1981. (Missouri Center for Health Statistics publication no 6.3).

5. Bjerkedal $\mathrm{T}$. Use of medical registration of birth in the study of occupational hazards to human reproduction. In: Hemminki K, Sorsa M, Vainio H, ed. Occupational hazards and reproduction. Hemisphere Publishing Co, Washington, DC 1985, pp 313-321.

6. Bjerkedal T. Occupation and outcome of pregnancy: A population-based study in Norway. In: Marois M, ed. Preventive physical and mental congenital defects, Part B: Epidemiology, early detection, therapy and environmental factors. Alan R Liss, Inc, New York, NY 1985, pp 265-268.

7. Blomqvist U, Ericson A, Kallen B, Westerholm P. Delivery outcome for women working in the pulp and paper industry. Scand J Work Environ Health 7 (1981) 114118.

8. Bracken MB. Methodologic issues in the epidemiologic investigation of drug-induced congenital malformations. In: Bracken MB, ed. Perinatal epidemiology. Oxford University Press, New York, NY 1984, pp 423-449.

9. Brent RL. The magnitude of the problem of congenital malformations. In: Marois M, ed. Prevention of physical and mental congenital defects, Part A: The scope of the problem. Alan R Liss, Inc, New York, NY 1985, pp 55-68.

10. Carucci PM. Reliability of statistical and medical information reported on birth and death certificates. New York State Department of Health, Albany, NY 1979. (New York State Department of Health monograph no 15).

11. Carucci PM, Prasad S. A comparison of mother's occupations reported on live birth certificates and on a sur- vey questionnaire. Public Health Rep 94 (1979) 432437.

12. Centers for Disease Control. Serum dioxin in Vietnamera veterans - Preliminary report. Morb Mortal Wkly Rep 36 (1987) 470-475.

13. Coggon D, Pannett B, Acheson ED. Use of a job-exposure matrix in an occupational analysis of lung and bladder cancers on the basis of death certificates. J Natl Cancer Inst 72 (1984) 61-65.

14. Cohen EN, Brown BW, Wu ML, Whitcher CE, Brodsky JB, Gift HC, Greenfield W, Jones TW, Driscoll EJ. Occupational disease in dentistry and chronic exposure to trace anesthetic gases. J Am Dent Assoc 101 (1980) $21-31$.

15. Cole P. The evolving case-control study. J Chronic Dis 32 (1979) $15-27$.

16. Corbett TH, Cornell RG, Endris JL, Lieding K. Birth defects among children of nurse-anesthetists. Anesthesiology 41 (1974) 341-344.

17. Council on Scientific Affairs. Effects of toxic chemicals on the reproductive system. J Am Med Assoc 253 (1985) $3431-3437$.

18. Edmonds LD, Anderson CE, Flynt JW, James LM. Congenital central nervous system malformations and vinyl chloride monomer exposure: A community study. Teratology 17 (1978) 137-142.

19. Edmonds LD, Layde PM, James LM, Flynt JW Jr, Erickson JD, Oakley GP Jr. Congenital malformations surveillance: Two American systems. Int J Epidemiol 10 (1981) 247-252.

20. Erickson JD, Cochran WM, Andersen CE. Parental occupation and birth defects, a preliminary report. Contrib Epidemiol Biostat 1 (1979) 107-117.

21. Erickson JD, Mulinare J, McClain PW, Fitch TG, James LM, McClearn AB, Adams MJ Jr. Vietnam veterans; risks for fathering babies with birth defects. J Am Med Assoc 252 (1984) 903-912.

22. Ericson A, Eriksson M, Kallen B, Westerholm P, Zetterstrom R. Delivery outcome of women working in laboratories during pregnancy. Arch Environ Health 39 (1984) 5-10.

23. Ericson A, Kallen B. Survey of infants born in 1973 or 1975 to Swedish women working in operating rooms during their pregnancies. Anesth Analg 58 (1979) 302-305.

24. Ericson A, Kallen B, Meirik O, Westerholm P. Gastrointestinal atresia and maternal occupation during pregnancy. J Occup Med 24 (1982) 515-518.

25. Gittelsohn AM, Milham S Jr. Vital record incidence of congenital malformations in New York State. In: Neel JV, Shaw MW, Schull WJ, ed. Genetics and the epidemiology of chronic diseases. Government Printing Office. Washington DC 1963, pp 305-319 (USPHS no 1163).

26. Grether JK. New California program monitors birth defects. J Perinatol 5 (1985) 8-10.

27. Hansson E, Jansa S, Wande H, Kallen B, Ostlund E. Pregnancy outcome for women working in laboratories in some of the pharmaceutical industries in Sweden. Scand J Work Environ Health 6 (1980) 131-134.

28. Hayden GF, Kramer MS, Horwitz RI. The case-control study: A practical review for the clinician. J Am Med Assoc 247 (1982) 326-331.

29. Hemminki K, Mutanen P, Luoma K, Saloniemi I. Congenital malformations by the parental occupation in Finland. Int Arch Occup Environ Health 46 (1980) 93-98.

30. Hemminki K, Mutanen P, Saloniemi I, Luoma K. Congenital malformations and maternal occupation in Finland: Multivariate analysis. J Epidemiol Community Health 35 (1981) 5-10.

31. Hemminki K, Saloniemi I, Luoma K, Salonen T, Partunen T, Vainio K, Hemminki E. Transplacental carcinogens and mutagens: Childhood cancer, malformations and abortions as risk indicators. In: Vainio $\mathrm{H}$, Sorsa M, Hemminki K, ed. Occupational cancer and car- 
cinogenesis. Hemisphere Publishing, Washington, DC 1980, pp 1115-1125.

32. Hewson D, Bennett A. Childbirth research data: Medical records or women's reports. Am J Epidemiol 125 (1987) 484-491.

33. Hoar SK, Morrison AS, Cole P, Silverman DT. An occupation and exposure linkage system for the study of occupational carcinogenesis. J Occup Med 22 (1980) $722-726$.

34. Holmberg PC. Central-nervous-system defects in children born to mothers exposed to organic solvents during pregnancy. Lancet 2 (1979) 177-179.

35. Holmberg PC, Hernberg S. Congenital defects and occupational factors: A comparison of different methodological approaches. Scand J Work Environ Health 5 (1979) 328-332.

36. Holmberg PC, Nurminen M. Congenital defects of the central nervous system and occupational factors during pregnancy: A case-referent study. Am J Ind Med 1 (1980) $167-176$.

37. Hook EB. Incidence and prevalence as measures of the frequency of birth defects. Am J Epidemiol 116 (1982) $743-747$.

38. Hook EB, Albright SG, Cross PK. Use of Bernoulli census and $\log$-linear methods for estimating the prevalence of spina bifida in livebirths and the completeness of vital records in New York State. Am J Epidemiol 112 (1980) 750-758.

39. Janerich DT, Polednak AP. Epidemiology of birth defects. Epidemiol Rev 5 (1983) 16-37.

40. Jick H, Vessey MP. Case-control studies in the evaluation of drug-induced illness. Am J Epidemiol 107 (1978) $1-7$.

41. Kallen B, Malmquist G, Moritz U. Delivery outcome among physiotherapists in Sweden: Is non-ionizing radiation a fetal hazard? Arch Environ Health 37 (1982) $81-85$.

42. Kalter H, Warkany J. Congenital malformations: Etiologic factors and their role in prevention (parts one and two). New Engl J Med 308 (1983) 424-431 \& 491-497.

43. Klemeti A, Saxen L. Prospective versus retrospective approach in the search for environmental causes of malformations. Am J Public Health 57 (1967) 2071-2075.

44. Klingberg MA, Papier CM, Hart J. Birth defects monitoring. Am J Ind Med 4 (1983) 309-328.

45. Knill-Jones RP, Rodrigues LV, Moir DD, Spence AA. Anaesthetic practice and pregnancy: Controlled survey of women anesthetists in the United Kingdom. Lancet 1 (1972) 1326-1328.

46. Knox EG, Armstrong EH, Lancashire R. The quality of notification of congenital malformations. $\mathbf{J}$ Epidemiol Community Health 38 (1984) 296-305.

47. Kurppa K, Holmberg PC, Hernberg S, Rantala K, Riala R, Nurminen T. Screening for occupational exposures and congenital malformations. Scand J Work Environ Health 9 (1983) 89-93.

48. Kurppa K, Holmberg PC, Rantala K, Nurminen T, Saxen L. Birth defects and exposure to video display terminals during pregnancy. Scand J Work Environ Health 11 (1985) 353-356.

49. Leffingwell SS, Robinson C, Lee ST, Ditzel C, Horning R, Cottrill C, Stephenson F, Halperin W. Report on women pharmaceutical workers and adverse reproductive outcomes. National Institute of Occupational Safety and Health, Cincinnati, OH 1983 (NTIS publication PB84-148956).

50. Lindbohm ML, Hemminki K, Kyyronen P, Kilpikari I, 'ainio $\mathrm{H}$. Spontaneous abortions among rubber workers and congenital malformations in their offspring. Scand J Work Environ Health 9 (1983) 85-90.

51. Linet MS, Stewart WF, Van Natta ML, McCaffrey LD, Szklo M. Comparison of methods for determining occupational exposure in a case-control interview study of chronic lymphocytic leukemia. J Occup Med 29 (1987) $136-141$.

52. Logue JN, Hamburger S, Silverman PM, Chiacchierini RP. Congenital anomalies and paternal occupational exposure to shortwave, microwave, infrared and acoustic radiation. J Occup Med 27 (1985) 451-452.

53. Mackeprang M, Hay S, Lunde AS. Completeness and accuracy of reporting of malformations on birth certificates. Health Serv Ment Health Adm Health Rep 87 (1972) 43-49.

54. Maternal and Child Health Branch, Epidemiological Studies Section, Department of Health Services. Congenital malformations reported on the California certificate of birth. Berkeley, CA 1982.

55. McDonald AD, Cherry NM, Delorme C, McDonald JC. Visual display units and pregnancy: Evidence from the Montreal survey. J Occup Med 28 (1986) 1226-1231.

56. Mitchell AA, Cottler LB, Shapiro S. Effect of questionnaire design on recall of drug exposure in pregnancy. Am J Epidemiol 123 (1986) 670-676.

57. National Institute for Occupational Safety and Health. National occupation hazard survey, 1972-74. Volumes 1-3. Cincinnati, OH 1978. (Department of Health and Human Services publications no $74-127,77-213$, 78-114).

58. Nishimura $H$, Tanimura T. Clinical aspects of the teratogenicity of drugs. Excerpta Med (1976) 84-86.

59. Nordstrom S, Beckman L, Nordenson I. Occupational and environmental risks in and around a smelter in Northern Sweden: VI Congenital malformations. Hereditas 90 (1979) 297-302.

60. Olsen J. Risk of exposure to teratogens amongst laboratory staff and painters. Dan Med Bull 30 (1983) 24-28.

61. Paganini-Hill A, Ross RK. Reliability of recall of drug usage and other health-related information: Implications for retrospective studies. Am J Epidemiol 116 (1982) $114-122$

62. Papier $\mathrm{CM}$. Parental occupation and congenital malformations in a series of 35,000 births in Israel. In: Marois $M$, ed. Prevention of physical and mental congenital defects, Part B: Epidemiology, early detection and therapy, and environmental factors. Alan Liss, Inc, New York, NY 1985, pp 291-294.

63. Pearn JH. Teratogens and the male: An analysis with special reference to herbicide exposure. Med J Aust 2 (1983) $16-20$.

64. Pershagen G, Axelson O. A validation of questionnaire information on occupational exposure and smoking. Scand J Work Environ Health 8 (1982) 24-28.

65. Pharoah POD, Alberman E, Doyle P, Chamberlain G. Outcome of pregnancy among women in anesthetic practice. Lancet 1 (1977) 34-36.

66. Polednak AP, Janerich DT. Uses of available record systems in epidemiologic studies of reproductive toxicology. Am J Ind Med 4 (1983) 329-348.

67. Roan CC, Matanoski GE, McIlnay CQ, Olds KL, Pylant F, Trout JR, Wheeler P, Morgan DP. Spontaneous abortions, stillbirths and birth defects in families of agricultural pilots. Arch Environ Health 39 (1984) $56-60$.

68. Saxen L. Drugs and teratogenesis. In: Colombo F, Shapiro S, Slone D, Tognoni G, ed. Epidemiological evaluation of drugs: Proceedings of the international symposium on epidemiological evaluation of drugs, Milan, Italy, PSG Publishing, Littleton, MA 1977, pp 267-276.

69. Saxen L. Twenty years of study of the etiology of congenital malformations in Finland. In: Kalter H, ed. Issues and reviews in teratology. Volume 1. Plenum Press, New York, NY 1984, pp 73-110.

70. Schlesselman JJ. Case-control studies. Oxford University Press, Oxford 1982.

71. Schulte PA. Methodologic issues in the use of biologic 
markers in epidemiologic research. Am J Epidemiol 126 (1987) 1006-1016.

72. Schwartz DA, Newsum LA, Heifetz R. Parental occupation and birth outcome in an agricultural community. Scand J Work Environ Health 12 (1986) 51-54.

73. Selevan SG, Lemasters GK. The dose-response fallacy in human reproductive studies of toxic exposures. J Occup Med 29 (1987) 451-454.

74. Shalat SL, Christiani DC, Baker EL. Accuracy of work history obtained from a spouse. Scand J Work Environ Health 13 (1987) 67-69.

75. Shepherd TH. Catalog of teratogenic agents. Fifth edition. Johns Hopkins University Press, Baltimore, MD 1986.

76. Siemiatycki J, Day NE, Fabry J, Cooper JA. Discovering carcinogens in the occupational environment: A novel epidemiologic approach. J Natl Cancer Inst 66 (1981) 217-225. (Guest editorial).

77. Smith AH, Fisher DO, Pearce N, Chapman CJ. Congenital defects and miscarriages among New Zealand 2,4,5-T sprayers. Arch Environ Health 37 (1982) $197-200$.

78. Smith AH, Matheson DP, Fisher DO, Chapman CJ. Preliminary report of reproductive outcomes among pesticide applicators using 2,4,5-T. New Z Med J 93 (1981) 177-179.

79. Snedecor GW, Cochran WG. Statistical methods. Seventh edition. Iowa State University Press, Ames, IA 1980.

80. Stein $\mathrm{Z}$, Kline $\mathrm{J}$, Kharrazi $\mathrm{M}$. What is a teratogen? Epidemiological criteria. In: Kalter $\mathbf{H}$, ed. Issues and reviews in teratology. Volume 2. Plenum Press, New York, NY 1983, pp 23-66.
81. Stellman JM. The occupational environment and reproductive health. In: Rom WN, ed. Environmental and occupational medicine. Little, Brown \& Co, Boston, MA 1983, pp 75-83.

82. Sullivan FM, Barlow SM. Congenital malformations and other reproductive hazards from environmental chemicals. Proc R Soc London (Biol) 205 (1979) 91-110.

83. Thomas DC, Siemiatycki J, Dewar R, Robins J, Goldberg M, Armstrong BG. The problem of multiple inference in studies designed to generate hypotheses. Am J Epidemiol 122 (1985) 1080-1095.

84. Tilley BC, Barnes AB, Bergstrath E, Labarthe D, Noller KL, Colton T, Adam E. A comparison of pregnancy history recall and medical records. Am J Epidemiol 121 (1985) 269-281.

85. Townsend JC, Bodner KM, Van Peenan PFD, Olson RD, Cook RR. Survey of reproductive events of wives of employees exposed to chlorinated dioxins. Am J Epidemiol 115 (1982) 695-713.

86. Wilson JG. Embryological considerations in teratology. In: Wilson JG, Warkany J, ed. Teratology principles and techniques. University Chicago Press, Chicago, IL 1965, pp 251-261.

87. Wilson JG, Fraser FC. Handbook of teratology. Volume 1 (General principles and etiology). Plenum Press, New York, NY 1977.

88. Zierler S, Rothman KJ. Congenital heart disease in relation to maternal use of Bendectin and other drugs in early pregnancy. N Engl J Med 313 (1985) 347-352.

Received for publication: 21 March 1988 